\title{
Does the Definition of Retirement Matter in Estimating the Effects of Retirement on Cognitive Functioning?
}

\author{
Shinya Kajitani ${ }^{a}, \underline{\text { Kei Sakata }}^{b}$, and Colin R. McKenzie ${ }^{c}$ \\ ${ }^{a}$ School of Economics, Meisei University, Tokyo, Japan \\ ${ }^{b}$ Faculty of Economics, Ritsumeikan University, Shiga, Japan \\ ${ }^{c}$ Faculty of Economics, Keio University, Tokyo, Japan \\ Email: ksakata@ec.ritsumei.ac.jp
}

\begin{abstract}
The purpose of this paper is to examine the causal impact of retirement on the cognitive functioning of male elderly workers using data from three waves of the National Survey of Japanese Elderly (NSJE). We examine how the definition of retirement affects the findings of Kajitani et al. (2013) where they examined the effects of the longest tenure job (career job) on cognitive functioning. Here, we use the status of retirement rather than the duration of retirement. The two step estimator we use takes account of the potential endogeneity of the status of retirement, using the age at which individuals are eligible to start receiving pension benefits and whether their career job was self-employment as instruments. Our empirical evidence suggests that the requirements in a person's career job have statistically significant impacts on the cognitive functioning after retirement. Kajitani et al.'s (2013) findings are found to be robust irrespective of the definition of retirement.
\end{abstract}

Keywords: Retirement, Cognitive Functioning, Occupation 
Kajitani et al., Does the definition of retirement matter in estimating the effects of retirement on cognitive functioning?

\section{INTRODUCTION}

How does work affect cognitive functioning? Does retirement induce a further decline in cognitive functioning? The relationship between retirement and cognitive skills has attracted much attention (Rohwedder and Willis, (2010) for a survey). These studies test the so-called 'use-it-or-lose-it' hypothesis, that is, they test for a causal effect of retirement on cognitive performance that suggests that following retirement cognitive performance declines because retired individuals do not use their cognitive skills as much as when they are working.

However, the empirical findings are not conclusive. Some find the evidence which is consistent with 'use-itor-lose-it' hypothesis (Rohwedder and Willis (2010), Bonsang et al. (2012)), whereas others do not (Coe and Zamarro (2011), Coe et al. (2012)). Moreover, previous studies have not adequately examined the effects of occupational heterogeneity on cognitive functioning. Coe et al. (2012) and Adams et al. (2006) examine such issues, but their occupational divisions are rather rough and simple (blue collar/ white collar or professional/ non-professional). Kajitani et al. (2013) investigate the effects of occupational heterogeneity on cognitive functioning by using much finer occupational decompositions using the Dictionary of Occupational Titles (DOT) by 3 digit occupational code, and find significant occupational effects. One of the contentious issues in this area is how we define retirement. The inconsistent findings of previous studies may be due to differences in the definition of retirement. This paper thus re-examines Kajitani et al.'s (2013) findings, and analyses how the change in the definition of retirement from duration of retirement to the status of the retirement affects their results. Retirement may just have one-off shock on cognitive functioning, and its duration may not be so important.

Following Kajitani et al. (2013), we examine the causal impact of retirement on cognitive functioning for elderly male workers using three waves of the "Longitudinal Study of a National Survey of Japanese Elderly" (NSJE), the initial 1987 survey, and two supplementary samples obtained in 1990, and 1996. We merge the occupational characteristics in the Dictionary of Occupational Titles (DOT) by 3 digit occupational code, and examine how occupational task requirements such as physical demands, mathematical development, reasoning development, and language development impact on cognitive functioning after retirement. Our two stage estimation technique deals with the potential endogeneity of retirement decisions by using two instruments: the age at which individuals are eligible to start receiving pension benefits and whether their career job was self-employment as instruments. The results are consistent with Kajitani et al (2013), and it may be argued that their results are robust.

The rest of this paper consists of four sections. Section 2 discusses the identification strategy and the models to be estimated, while section 3 describes the data. Section 4 reports the results of estimation and discusses their implications, and section 5 contains a brief conclusion.

\section{EMPIRICAL MODEL AND IDENTIFICATION ISSUES}

The main goal of this paper is to identify the causal effect of retirement on cognitive functioning and to examine whether aspects of the career job can have any effects on cognitive functioning after retirement. The following model is considered:

$C O G_{i}=\tau_{1} R E T_{i}+\tau_{2}\left(R E T_{i} \cdot D O T J_{i}\right)+\tau_{3} D O T J_{i}+X_{i} \beta+u_{i}$

where $C O G_{i}$ is the outcome variable (cognitive test score), $R E T_{i}$ is retirement variable, $D O T J_{i}$ is a $0-1$ dummy variable relating to the $J$ th occupational characteristics of the respondent's career job (longest job), $X_{i}$ is a vector of control variables, which includes a constant, the respondent's age and the respondent's years of education, $u_{i}$ is an error term, and the subscript $i$ refers to the $i$ th individual. Following Roberts and Binder's (2009) suggestion, we also include 1990 and 1996 survey year dummies in $X_{i}$ to account for the fact that we are combining information from three surveys, the 1987, 1990 and 1996 surveys. The use it or lose it hypothesis suggests that retirement should induce a decline in cognitive functioning, that is, $\tau_{1}<0$.

For $R E T_{i}$ in equation (1), this paper uses the status of retirement. We use four occupational characteristics in the Dictionary of Occupational Titles (DOT) of the respondent's career job, namely physical demands, mathematical development, reasoning development and language development. For the physical demands characteristic, we create a 0-1 dummy variable which takes the value unity if the score for the respondent's characteristic is higher than the median value, and zero otherwise. For other characteristics, we create a 0-1 dummy variable which takes the value unity if the score for the respondent's characteristic is higher than the median value, and zero otherwise. We label these variables DOTP for physical demands, DOTM for 
Kajitani et al., Does the definition of retirement matter in estimating the effects of retirement on cognitive functioning?

mathematical development, DOTR for reasoning, and DOTL for language development. We will include these four DOT variables one by one in equation (1). When the occupational characteristic relates to the physical demands of a job, a value of the dummy variable equal to 1 (0) relates to high (low) physical demands, so it is expected that $\tau_{3}<0$. For the other three variables, where a higher than median value corresponds to a value of the dummy variable equal to unity, we also expect that $\tau_{3}>0$. For the interaction term $R E T_{i} \cdot D O T J_{i}$, the rate of decline of cognitive function with retirement will be higher for job careers that involve higher than the median physical demand characteristics, so it is also expected that $\tau_{2}<0$. For the other three variables, it is expected that $\tau_{2}>0$.

The possibility that the status of retirement in equation (1) is endogenous is a major obstacle to estimating the causal impact of retirement on cognitive functioning. Individuals whose cognitive abilities are lower (higher) may retire earlier (later). Moreover, retirement choices may be correlated with unobservable factors such as health. The typical identification strategy in previous studies is to use changes in the social security system as an instrument for the retirement variable, and we also adapt this strategy.

We consider the following model to explain the choice of whether to retire:

$R E T_{i}^{*}=\gamma_{1}$ PENSION $_{i}+\gamma_{2}$ SELF $_{i}+\gamma_{3}$ DOTJ $_{i}+X_{i} \delta+w_{i}$

$R E T_{i}=\left\{\begin{array}{l}1 \text { if } R E T_{i}^{*}>0 \\ 0 \text { if } R E T_{i}^{*} \leq 0\end{array}\right.$

where $R E T_{i}^{*}$ is an unobserved latent variable which is connected to the observed $R E T_{i}$ by equation (3), $\operatorname{PENSION}_{i}$ is the age at which individual $i$ is eligible to start receiving pension benefits, $S E L F_{i}$ is a $0-1$ dummy variable taking the value unity if the individual $i$ 's career job is a self-employed job, $X_{i}$ is the same vector of control variables as used in equation (1), and $w_{i}$ is a disturbance which is assumed to be normally independently and identically distributed with zero mean and unit variance. The combination of (2) and (3), together with the assumptions about $w_{i}$ means that this model can be estimated using the Probit technique.

By comparing equations (1) and (2), it can be seen that our "instruments" for $R E T_{i}$ are the age at which an individual is eligible to start receiving pension benefits, $\operatorname{PENSION}_{i}$, and the self-employed dummy variable, $S E L F_{i}$. The age at which individuals are eligible to start receiving pension benefits has changed over the years in Japan. Due to amendments to the pension law in 1954, the age at which men are eligible to start receiving pension benefits was gradually raised by one year in every four years from 55 to 60 over a period of 16 years, 1957 to $1973^{1}$. As a result, men born from 2 May 1902 to 1 May 1905 were eligible to start receiving their pension benefits at the age of 56. Similarly, men born from 2 May 1905 to 1 May 1908 were eligible to start receiving their pension benefits at the age of 57, and so on. Men born after 2 May 1914 were eligible to start receiving their pension benefits at the age of 60 . We assume that these are exogenous changes to the timing of pension benefits, so that the changes in the eligibility age to receive pension benefits is only correlated with the retirement variable, but not with cognitive functioning.

The second instrument is a self-employment dummy which controls for whether the respondent's career job was self-employment. It is important to note that the self-employed dummy refers to whether or not the respondent's career job was self-employment, not whether or not the respondent's job immediately prior to retirement was self-employment. This variable aims to capture differences in the pension system for different types of workers. Self-employed workers are covered by the National Pension System (Kokumin Nenkin), whereas employed workers are covered by the Employees' Pension Insurance System (Kousei Nenkin Hoken). We expect these schemes have different effects on the timing of retirement.

In order to estimate the parameters of equation (1) taking account of the endogenity of the status of retirement, we employ a two step estimator. First, we estimate the parameters in equations (2) using a Probit estimator to obtain estimates of the parameters of $\gamma_{i}$ and $\delta, \widehat{\gamma}_{1}$ and $\hat{\delta}$, respectively. From equations (2) and (3), the conditional expectation of $R E T_{i}$ can be computed, and then, it was inserted in equation (1) instead of $R E T_{i}$

\section{DATA}

Our data are drawn from the 1987 (Wave 1), 1990 (Wave 2), and 1996 (Wave 4) waves of the "Longitudinal Study of a National Survey of Japanese Elderly (NSJE)" (Zenkoku Koureisha no Seikatsu to

\footnotetext{
${ }^{1}$ It should be noted that this law did not change the age at which women were eligible to start receiving their pension benefits. This is another reason why we did not include females in our analysis.
} 
Kajitani et al., Does the definition of retirement matter in estimating the effects of retirement on cognitive functioning?

Kenkounikansuru Chouki Jyuudann Chousa). For the purpose of this survey, the "elderly" are defined as people who are 60 years of age or over. These surveys were conducted by the Tokyo Metropolitan Institute of Gerontology and the University of Michigan. The data was provided by the Social Science and the Social Science Japan Data Archive, Information Center for Social Science Research on Japan, Institute of Social Science, the University of Tokyo. NSJE has been conducted every three years since 1987.The population of aged 60 and over was extracted by two-stage stratified random sampling. The surveys include information on the respondent's physical health, mental health, family relationships, social relationships, and economic status. Observations where for health reasons a family member answered the survey on behalf of the respondent are excluded from our analysis. It is important to note that these observations do not contain not only the memory test scores, but also information on the timing of retirement. Thus, we could not include these extreme cases of deterioration of cognitive function into our analysis. Kan (2009) and Kajitani (2011) compare the differences between the sample distributions of the NSJE and the relevant Japanese Census data, and report that there is little difference between the two.

The analysis in this paper is restricted to males. In Japan, women are more likely to quit their jobs after marriage and child birth than in other developed countries. Some women come back to work after child birth, and others do not. The NSJE survey does not provide information on how long respondents are away from their jobs and at what age they returned to the workforce. More importantly, for females in these generations in the sample, in particular, a sizeable number of individuals never worked. These women called "Kaji Testudai" in Japanese help with domestic work in their parental home until they get married and then become full time housewives after their marriage. For these reasons, the analysis of job effects on cognitive functioning for females could be a little more complicated than the analysis for males.

The supplementary sample in Wave 2 (1990) contains an additional 580 individuals who are aged 60 to 62 obtained by the stratified random sampling in addition to those individuals continuing from Wave 1 . In Wave 4 (1996), an additional 1210 individuals who are aged 60 to 65 obtained by the stratified random sampling are added to those continuing from Wave 3. Using the supplementary samples in Waves 2 and 4 in addition to the original sample in Wave 1 means that individuals who are in their early 60s are over represented in the sample we use. In order to adjust the age representation in the sample, we use weights from the Census when estimating models at both the first and second stages. All the descriptive statistics and estimation results reported in this paper weight the data appropriately to take account of this over representation. The analysis in this paper is restricted to males. All data on the variables used in this paper are drawn from NSJE except the Dictionary of Occupational Titles (DOT).

\subsection{Cognitive Test Scores}

NSJE contains information on the respondents' answers to questions that test his/her memory. In an interview, the respondent is asked nine questions: 1) the respondent's address; 2) the date of the interview; 3 ) the day of the interview 4) the respondent's mother's maiden name; 5) the name of the current Prime Minister; 6) the name of the previous Prime Minister; 7) the respondent's date of birth; 8) the respondent's age; and 9) a question that requires the respondent to continuously deduct 3 from 20 . We use the accuracy of the respondent's answers to these questions as a measure of cognitive functioning. For the question on successively deducting 3 from 20, the answer is recorded as being correct only if the respondent could successfully deduct 3 six times until the number becomes 2 . Over 80 percent of the respondents gave the correct answer to this question. This memory test is similar to standard memory tests in cognitive science (for example, Teng et. al, 1994).

We create a cognitive score variable based on seven of the nine questions which excludes the accuracy of answers to the questions about the current and the previous Prime Ministers because these two questions relating to the Prime Minister do not necessarily capture the respondent's memory loss. There were eight Prime Ministers within the sample period (1987-1996), and it is hard to identify if the wrong answer means a memory loss or a low interest in politics.

For each of the remaining seven questions, a correct answer to a question is allocated one point and an incorrect answer is allocated zero points, so the maximum possible score for an individual is 7 and the minimum possible score is 0 .

\subsection{Retirement}

Previous studies such as Bonsang et al. (2010) and Rohwedder and Wills (2010) use a retirement dummy, which takes the value unity if the respondent reports he/she is not working, and the value zero if s/he reports 
Kajitani et al., Does the definition of retirement matter in estimating the effects of retirement on cognitive functioning?

to be currently working for pay. Here, we take the same approach to check the robustness of Kajitani et al (2013) where the duration of retirement is used instead of the status of retirement.

\subsection{Dictionary of Occupational Titles (DOT)}

One main objective is to investigate how occupational characteristics of a respondent's career job are associated with cognitive functioning after retirement. We merge the 3 digit occupation codes in the Dictionary of Occupational Titles (DOT) with our data set using the 3 digit occupation code (288 occupations) in the NSJE. We examine four dimensions of an occupational task, namely, physical demands, mathematical development, reasoning development, and language development.

Following Fletcher et al (2011), in the physical demands category we focus on strength, which is measured as one of the five categories: Secondary, Light, Medium, Heavy and Very Heavy. We compute the physical strength variable by giving scores as follows: Secondary $=1$, Light $=2$, Medium $=3$, Heavy $=4$ and Very Heavy $=5$.

DOT also provides information of the General Educational Development (GED) associated with occupations. It describes the levels of educational aspects which are required of the worker for satisfactory job performance. There are three aspects of GED: mathematical development, reasoning development, and language development. Each of these GED variables is measured on a scale of 1 to 6 . We merge this information using the occupational code in NSJE and examine the depth of white-collar-type work in the worker's career job. For each NSJE code we compute the average score for physical demands, mathematical development, reasoning development, and language development for the DOT codes corresponding to this category. We then create a dummy variable for each of the four characteristics which take the value unity if the score of characteristics in the respondent's NSJE occupation is higher than the median value, and 0 otherwise. We will include DOT variables one by one in equation (1). Descriptive statistics on all the variables used in the analysis are reported in Table 1.

Table 1: Descriptive Statistics (Males)

\begin{tabular}{|c|c|c|c|c|}
\hline \multirow{2}{*}{ With weighting } & \multicolumn{4}{|c|}{ Sample size $(1,177)$} \\
\hline & Mean & Std. Dev. & Min & $\operatorname{Max}$ \\
\hline Memory test score & 6.730 & 0.586 & 2 & 7 \\
\hline Retirement ( 1 if the respondent was retired) & 0.441 & 0.497 & 0 & 1 \\
\hline Physical demands ( 1 if $>$ median) & 0.498 & 0.500 & 0 & 1 \\
\hline Mathematical development ( 1 if $>$ median) & 0.513 & 0.500 & 0 & 1 \\
\hline Reasoning development ( 1 if $>$ median $)$ & 0.503 & 0.503 & 0 & 1 \\
\hline Language development ( 1 if $>$ median $)$ & 0.504 & 0.500 & 0 & 1 \\
\hline Age (years) & 67.375 & 6.456 & 60 & 87 \\
\hline Education (year) & 9.889 & 2.942 & 0 & 17 \\
\hline Age eligible for pension benefit (year) & 59.559 & 1.035 & 55 & 60 \\
\hline Self-employed ( 1 if career job=self-employed) & 0.417 & 0.493 & 0 & 1 \\
\hline year 1987 ( 1 if the 1987 survey is used) & 0.692 & 0.462 & 0 & 1 \\
\hline year 1990 ( 1 if the 1990 survey is used) & 0.061 & 0.239 & 0 & 1 \\
\hline
\end{tabular}

Source: Authors calculations using data from NSJE.

\section{RESULTS AND DISCUSSION}

All estimates reported in this paper are obtained using STATA version 11. Due to space limitations, results for the estimating the first stage estimation are not reported. However, they show that our instruments, the age at which an individual is eligible to start receiving pension benefits and the self-employed dummy are both statistically significant. The models estimated all pass the tests for weak instruments. Table 2 reports the second stage estimates, and show that the status of retirement is not statistically significant except for (2) in Table 2, which is positive and contradicts previous studies. However, the results in the other cases are all consistent with some of the previous studies. 
Kajitani et al., Does the definition of retirement matter in estimating the effects of retirement on cognitive functioning?

Table 2: Estimated Results: Two Staged Estimation (With weighting)

Cognitive equation

\begin{tabular}{|c|c|c|c|c|c|c|c|c|}
\hline & (1) & & (2) & & (3) & & (4) & \\
\hline \multirow[t]{2}{*}{ RET (fitted value) } & -0.214 & & 0.306 & $*$ & 0.227 & & 0.247 & \\
\hline & {$[0.164]$} & & {$[0.175]$} & & {$[0.174]$} & & [0.169] & \\
\hline \multirow[t]{2}{*}{ RET (fitted value)*DOTP } & 0.413 & $* *$ & & & & & & \\
\hline & {$[0.188]$} & & & & & & & \\
\hline \multirow[t]{2}{*}{ RET (fitted value)*DOTM } & & & -0.610 & $* * *$ & & & & \\
\hline & & & {$[0.198]$} & & & & & \\
\hline \multirow[t]{2}{*}{ RET (fitted value)*DOTR } & & & & & -0.448 & $* *$ & & \\
\hline & & & & & {$[0.204]$} & & & \\
\hline \multirow[t]{2}{*}{ RET (fitted value)*DOTL } & & & & & & & -0.492 & $* *$ \\
\hline & & & & & & & {$[0.203]$} & \\
\hline \multirow[t]{2}{*}{ DOTP } & -0.123 & & & & & & & \\
\hline & {$[0.082]$} & & & & & & & \\
\hline \multirow[t]{2}{*}{ DOTM } & & & 0.215 & $* *$ & & & & \\
\hline & & & {$[0.095]$} & & & & & \\
\hline \multirow[t]{2}{*}{ DOTR } & & & & & 0.165 & $*$ & & \\
\hline & & & & & {$[0.100]$} & & & \\
\hline \multirow[t]{2}{*}{ DOTL } & & & & & & & 0.169 & $*$ \\
\hline & & & & & & & {$[0.097]$} & \\
\hline Sample size & 1,177 & & 1,177 & & 1,177 & & 1,177 & \\
\hline R-squared & 0.038 & & 0.045 & & 0.038 & & 0.040 & \\
\hline Wald test & 20.39 & $* * *$ & 28.69 & $* * *$ & 21.28 & $* * *$ & 22.45 & $* * *$ \\
\hline
\end{tabular}

Notes: 1) Robust standard errors in brackets; 2) *,** and *** indicate statistical significance at $10 \%, 5 \%$ and $1 \%$ levels respectively; and 4) the Wald tests the null hypothesis that the coefficients for all variables except the constant are jointly zero; 4) full results available upon request.

Furthermore, the cross term variable for physical demand is also negative and statistically significant, and the cross terms for mathematical development, reasoning development and language development are also positive and statistically significant. It should be noted that in none of the estimated results were the survey dummies ever significant in ether the first or second stage suggesting that it is legitimate to combine the information from different surveys with appropriate weights.

Our empirical findings highlight that the requirements of a person's career job have statistically significant impacts on cognitive functioning after retirement. Due to potential multicollinearity, we could not include all of the job characteristic variables in one equation. Career jobs with high physical demands reduce the memory test score after retirement. In contrast, career jobs with high mathematical development, reasoning development and language development are important in preventing the decline of cognitive functioning after retirement. Overall, the results are similar to those reported in Kajitani et al. (2013). Thus, it can be argued that Kajitani et al.'s (2013) results are robust irrespective of the definition of retirement.

\section{CONCLUSION}

This paper examines the causal impact of retirement on cognitive functioning for elderly male workers in Japan using data from several waves of the National Survey of Japanese Elderly (NSJE). We conducted a robustness check of Kajitani et al. (2013). Kajitani et al. (2013) use the duration of retirement as their retirement variable, and they explore the effects of the characteristics of the longest tenured job (career job) on cognitive functioning. Our empirical evidence suggests that if the individual's career job requirement has high mathematical development, reasoning development, and language development, the memory loss after retirement is slower. On the other hand, physical task performed in an individual's career job increases the deterioration of memory loss after retirement. Such results are consistent with Kajitani et al. (2013), and the change in the definition of retirement from the duration of retirement to the status of retirement did not alter the overall findings of Kajitani et al. (2013). 
Kajitani et al., Does the definition of retirement matter in estimating the effects of retirement on cognitive functioning?

There are of course some reservations concerning the interpretation of our results even when we conducted the robustness check. Although we found slower deterioration in the memory loss among people who engaged in the job tasks with a higher level of mathematical development, reasoning development, and language development, this may not mean such activities stimulate brain and delay the pace of deterioration of memory loss. One alternative interpretation is that people who worked in such occupations may have some specific patterns in how they spend time in their after-retirement life. In this case, occupational tasks may not have direct impacts on cognitive functioning. This issue should be dealt with in further studies.

\section{ACKNOWLEDGMENTS}

All three authors would like to thank participants at a Workshop on Family Economics at the University of Bocconi where an earlier version of this paper was presented, and would like to gratefully acknowledge the financial assistance provided by the Japan Society for the Promotion of Science (JSPS) Grant in Aid for Scientific Research (B) No. 24330093 for a project on "Retirement Behavior of the Aged and their Cognitive Ability and Health". They would also like to acknowledge the kind permission of the Tokyo Metropolitan Institute of Gerontology, the University of Michigan and the Social Science and the Social Science Japan Data Archive, Information Center for Social Science Research on Japan, Institute of Social Science, University of Tokyo in making available the data in the "'Longitudinal Study of a National Survey of Japanese Elderly (NSJE)" (Zenkoku Koureisha no Seikatsu to Kenkou ni kansuru Chouki Jyuudann Chousa) for use in the analysis in this paper. The third author wishes to gratefully acknowledge the research support provided by the Adolfo Ibanez University, Sciences Po, and the University of Bocconi.

\section{REFERENCES}

Adam S., Bay C., Bonsang E., Germain S., \& Perelman S. (2006). Occupational Activities and Cognitive Reserve: A Frontier Approach Applied to the Survey on Health, Ageing, and Retirement in Europe (SHARE). CREPP Working Paper No. 2006/05, Centre de Recherche en Economie Publique et de la Population (CREEP), Belgium.

Bonsang E., Adam S. \& Perelman S. (2012). Does Retirement Affect Cognitive Functioning? Journal of Health Economics, 31, 490-501.

Coe N.B., von Gaudecker H.M., Lindeboom M. \& Maurer J. (2012). The Effect of Retirement on Cognitive Functioning, Health Economics, 21(8), 913-927.

Coe N.B. \& Zamarro G. (2011). Retirement Effects on Health in Europe, Journal of Health Economics, 30 (1), 77-86.

Fletcher J.M., Sindelar J.L. \& Yamaguchi S. (2011). Cumulative Effects of Job Characteristics on Health. Health Economics, 20(5), 553-570.

Kajitani S. (2011). Working in Old Age and Health Outcomes in Japan, Japan and the World Economy, 23(3), 153-162.

Kajitani S., Sakata K., \& McKenzie C. (2013). Occupation, Retirement, and Cognitive Functioning, Joint Research Center for Panel Studies Discussion Paper Series DP2012-002.

Kan M. (2009). Economic Analysis of Disparities in Health among Japanese Elderly, Japanese Journal of Health Economics and Policy, 20(2), 85-108 (in Japanese).

Mazzonna F. \& Peracchi F. (2012). Ageing, Cognitive Abilities and Retirement. European Economic Review, 56(4), 691-710.

Roberts G. \& Binder D. (2009). Analyses Based on Combining Similar Information from Multiple Surveys. Paper Presented at the Survey Research Methods, Section of the Joint Statistical Meetings (JSM), Washington, DC. Accessed 27 January 2013. Available from <http://www.amstat. org/sections/srms/Proceedings/y2009/Files/303934.pdf>.

Rohwedder S. \& Willis R.J. (2010). Mental Retirement, Journal of Economic Perspectives, 24(1), 119-138.

Teng E. L., Hasegawa K., Homma A., Imai Y., Larson E., Graves E., Sugimoto K., Yamaguchi T., Sasaki T., Chiu D., \& White L. R. (1994). The Cognitive Abilities Screening Instrument (CASI): A Practical Test for Cross-Cultural Epidemiological Studies of Dementia, International Psychogeriatrics, 6(1), 45-58. 